\title{
SYNTHESIS OF FUNCTIONALIZED MCM-41 MESOPOROUS SILICA
}

\author{
Nevin Karamahmut Mermer, ${ }^{1}$ Muge Sari Yilmaz ${ }^{2}$
}

\begin{abstract}
The invention of mesoporous materials is of significant interest to many scientists worldwide. The Mobil Crystalline Materials No 41 (MCM-41) is a well-known mesoporous molecular sieve that was discovered in 1992 by a scientist at the Mobil Oil Corporation. The MCM-41 is widely used in catalysis, ion exchange, drug delivery, optics, gas sensing, and sorption. In this study, the surface of a mesoporous silica MCM-41, synthesized from pure silica, is functionalized with a methyl group by grafting. The synthesized and functionalized samples are characterized by X-ray powder diffractometer (XRD), and the functionalized sample are also characterized by Fourier transform infrared spectroscopy (FTIR). The textural properties of the samples are determined using $\mathrm{N}_{2}$ adsorption and desorption analysis. Thermal behaviors of the samples are analyzed using thermogravimetry (TG) and derivative thermogravimetry (DTG). The results of the analyses show that the functionalization of the synthesized material through grafting was accomplished with the surface area of the functionalized sample determined as $600.87 \mathrm{~m}^{2} \mathrm{~g}^{-1}$.
\end{abstract}

UDC Classification: 66.03; DOI: http://dx.doi.org/10.12955/cbup.v5.1089

Keywords: MCM-41, grafting, methyl group, functionalization, XRD

\section{Introduction}

The Mobil Crystalline Materials No 41 (MCM-41) is a member of the M41S family and was first discovered by researchers at the Mobil Research and Development Corporation in 1992 (Gaydhankar et al., 2006; Feil et al., 2009). Porous materials can be classified based on certain criteria, such as pore shape, pore size, and production method. The most widely accepted classification is that of the International Union of Pure and Applied Chemistry (IUPAC) using pore sizes: microporous (pore diameter $<2 \mathrm{~nm}$ ), mesoporous (pore diameter $2-50 \mathrm{~nm}$ ), and macroporous (pore diameter $>50 \mathrm{~nm}$; Kenneth, 1998).

The MCM-41 is an ordered mesoporous hexagonal structure that has an increasing number of applications, such as adsorption, optics, gas sensing, catalysis, drug delivery systems, molecular sieving, and membranes for filtration and ion exchange (Belmabkhout et al., 2009; Kim et al., 1995; Kozhevnikov 1995; Selvam et al., 2001; Xiao-Dong et al., 2010; Yuliarto et al., 2004; Zhao et al., 1996). It is an ideal candidate for specific adsorbent design because the pore size can be adjusted by modifying or functionalizing the organic functional groups that are used in quite extensive experimental investigations (Araki et al., 2009; Vartuli et al., 1996). Functionalization with organic and inorganic groups is carried out to improve the physical and chemical properties of mesoporous silica materials. These new organic-inorganic hybrid mesoporous silica structures have attracted attention due to their large surface areas, pore structures, and functionalized structures. These properties avail its extensive use in several areas of application (Taib et al., 2011). Studies on using mesoporous silica modified with amine groups as adsorbents in $\mathrm{CO}_{2}$ adsorption are quite extensive (Belmabkhout et al., 2010; Builes \& Vega, 2012; Gholami et al., 2016; Klinthong et al., 2013; Liu et al., 2015; Wang et al., 2015). The various chemical ligands that are either organic or inorganic can be anchored on the surface of MCM-41. These modifications have a considerable effect on the surface and structural properties of the mesoporous materials (Zhao et al., 2000). For instance, silylation of the MCM-41 improves the hydrothermal and mechanical stability of material due to its advanced hydrophobicity (Zhao \& Lu, 1998). There are studies that attract great interest regarding the application of the functionalized mesoporous materials by organic compounds. These include heavy metal removal from water, $\mathrm{CO}_{2}$ capturing, drug delivery system, and catalytic applications (Builes et al., 2012; Faghihian \& Naghavi, 2014; Manzano et al., 2008; Rath et al., 2014; Wu et al., 2010). In the present study, the MCM-41 mesoporous silica was synthesized from sodium metasilicate pentahydrate, and then, modified using tri-methoxymethyl-silane (TMMS). The synthesized pure sample was characterized by X-ray powder diffraction (XRD), $\mathrm{N}_{2}$ adsorption and

\footnotetext{
${ }^{1}$ Faculty of Chemical and Metallurgical, Yildiz Technical University, nevinkaramahmut@ hotmail.com

${ }^{2}$ Faculty of Chemical and Metallurgical, Yildiz Technical University, mugesari@yildiz.edu.tr
} 
desorption, and DTA or TG. The functionalized sample was also characterized by XRD, Fourier transform infrared spectroscopy (FTIR), $\mathrm{N}_{2}$ adsorption and desorption, and DTA or TG.

\section{Data and Methodology}

The pure silica source used in the synthesis of MCM- 41 was sodium metasilicate pentahydrate (SMP, $\mathrm{SiO}_{3} \mathrm{Na}_{2} .5 \mathrm{H}_{2} \mathrm{O}, 99 \%$ purity). The organic template hexadecyltrimethylammonium bromide (HTABr, $99 \%$ purity), sodium hydroxide $(\mathrm{NaOH})$, sulfuric acid $\left(\mathrm{H}_{2} \mathrm{SO}_{4}, 95-98 \%\right)$, trimethoxymethylsilane (TMMS), $\left.\mathrm{CH}_{3} \mathrm{Si}\left(\mathrm{OCH}_{3}\right)_{3}, \geq 98.0 \%\right)$, and toluene $\left(\mathrm{C}_{6} \mathrm{H}_{5} \mathrm{CH}_{3}, \geq 99.9 \%\right)$ were obtained from Merck.

In the functionalization process, the sample was mixed in the Zhicheng ZHWY-200B brand incubator shaker. Crystallographic properties of the synthesized samples were investigated using a PANalytical X'Pert-Pro XRD instrument with $\mathrm{Cu}-\mathrm{K} \alpha$ tube $(\lambda=0.153 \mathrm{~nm})$. The functional band of the modified sample was determined by the FTIR-KBr technique with Perkin Elmer Spectrum One FT-IR spectrophotometer at a wavelength of $400-450 \mathrm{~cm}^{-1}$.

Textural properties of the samples were determined on a Micromeritics ASAP 2020 surface area and porosimetry system. The specific surface area of the synthesized samples was calculated using the Brunauer-Emmett-Teller (BET) method. The BET surface area $\left(\mathrm{S}_{\mathrm{BET}}, \mathrm{m}^{2} \mathrm{~g}^{-1}\right)$ was calculated using the adsorption data, with a relative equilibrium pressure $\left(\mathrm{P} / \mathrm{P}_{0}\right)$ in the range of 0.03 to 0.30 .

Thermal properties of synthesized and modified MCM-41samples were subjected to thermal analysis in a Perkin Elmer Pyris Diamond DTA-TG thermogravimetry instrument. Analyses of the samples were carried out at nitrogen flow rate of $200 \mathrm{ml} \mathrm{min}^{-1}$. The samples were heated from $35^{\circ} \mathrm{C}$ to $900{ }^{\circ} \mathrm{C}$ with a heating rate of $10^{\circ} \mathrm{C} \mathrm{min}^{-1}$ by using a platinum crucible.

The Synthesis of MCM-41

For the synthesis of MCM-41, a certain amount of SMP was dissolved in water to prepare 1.5-M sodium silicate solution. The appropriate amount of sodium silicate solution was added drop by drop into the prepared $\mathrm{HTABr}$ solution, and then, the obtained solution was stirred for one hour with a magnetic stirrer. The $\mathrm{pH}$ of the solution was adjusted to 11 with sulfuric acid. After the $\mathrm{pH}$ adjusting, the solution was aged overnight. The solution with the precipitate was filtered and washed with distilled water. The resulting white-solid sample was dried overnight at $100{ }^{\circ} \mathrm{C}$ and then, calcined at $550{ }^{\circ} \mathrm{C}$ to remove the template from the structure.

\section{Functionalization of Obtained MCM-41}

Functionalization of the synthesized sample with methyl groups was carried out by a grafting method. Firstly, a certain amount of MCM-41 sample was dissolved in dry toluene, then TMMS was added to the mixture under stirring. The resulting mixture was added to an incubator shaker and stirred at room temperature for 24 hours. At the end of the shaking period, the solution was left under reflux for six hours. The mixture was filtered, washed several times with dry toluene, and then dried at room temperature. The functionalized sample was named MCM-41-TMMS.

\section{Results and Discussion}

Figure 1 presents the XRD diagrams of prepared MCM-41-TMMS and MCM-41 adsorbents. The diagrams show characteristic peaks of MCM-41, namely, a strong (100) reflection peak with two small peaks (110 and 200) resulted after functionalization. This result indicates that the regular structure of MCM-41 was preserved after functionalization. In comparison, the peak intensities of MCM-41TMMS (MCM-41 after the methyl group grafting) were noticeably lower.

The FTIR spectrum of the MCM-41-TMMS is shown in Figure 2. Only the peaks that differed from the MCM-41 sample were marked in the spectrum. Accordingly, the observed peaks at 2974.13 and $2922.41 \mathrm{~cm}^{-1}$ belonged to the $\mathrm{C}-\mathrm{H}$ asymmetric stretching vibrations of the methyl groups. The symmetric stretching vibration of $\mathrm{C}-\mathrm{H}$ appeared at $2853.44 \mathrm{~cm}^{-1}$. The observed peak at $1277.06 \mathrm{~cm}^{-1}$ was affiliated to the symmetric deformation bending of $\mathrm{C}-\mathrm{H}$. The FT-IR spectrum of MCM-41TMMS indicates whether a methyl group has been successfully grafted on the MCM-41 structure (AlOweini \& El-Rassy, 2009). 


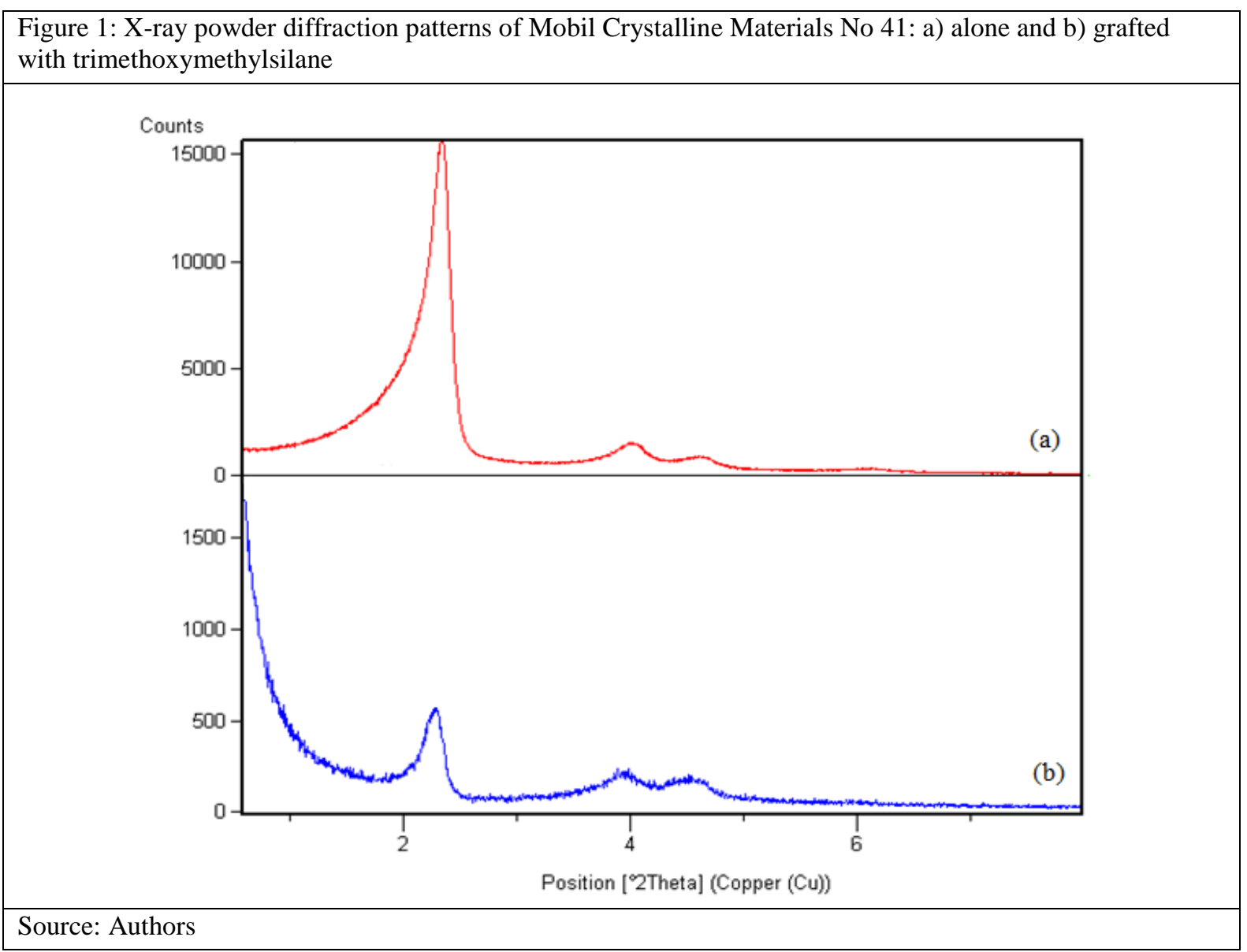

Figure 2: The Fourier transform infrared plot of Mobil Crystalline Materials-41 grafted with trimethoxymethylsilane

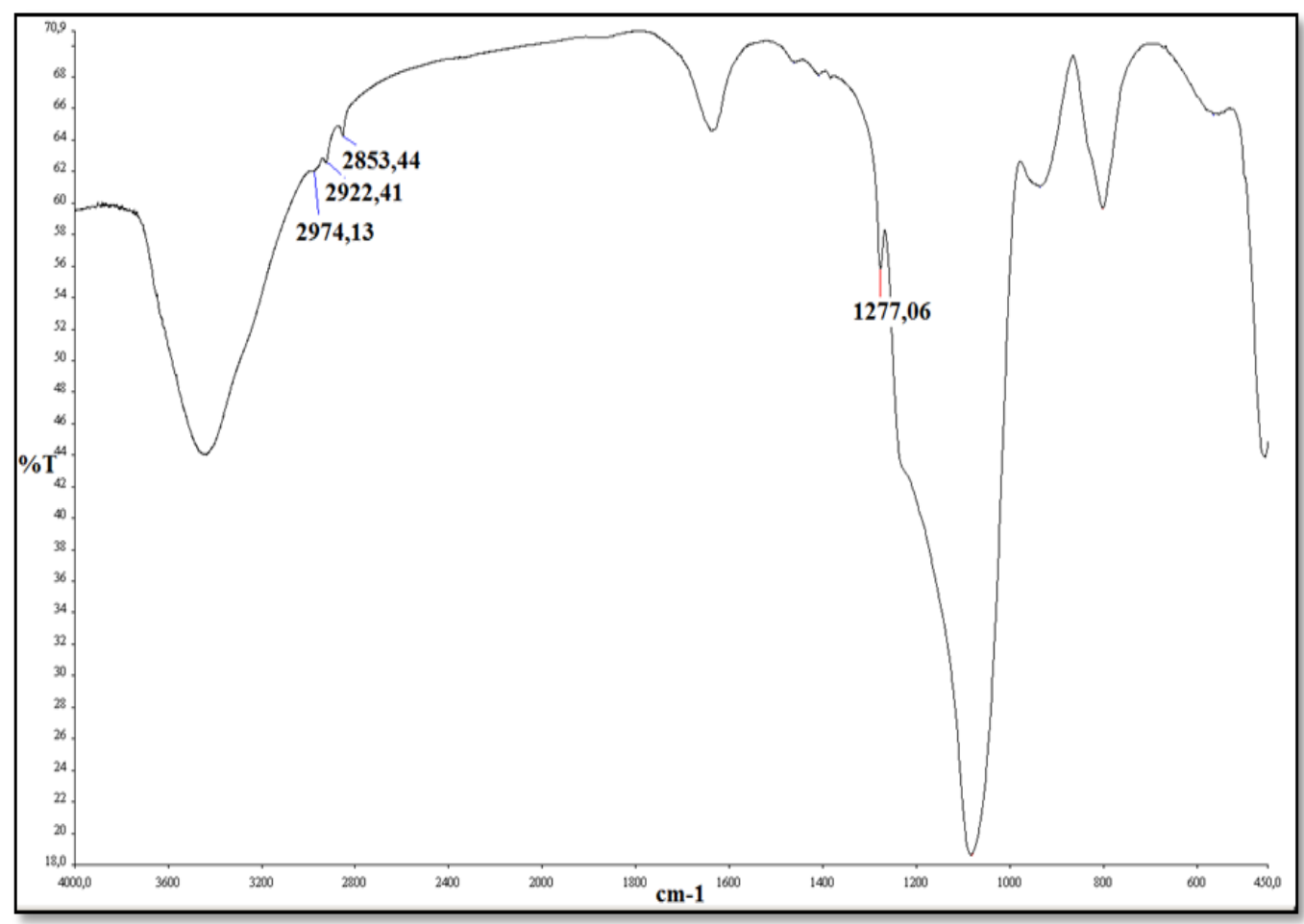

Source: Authors 


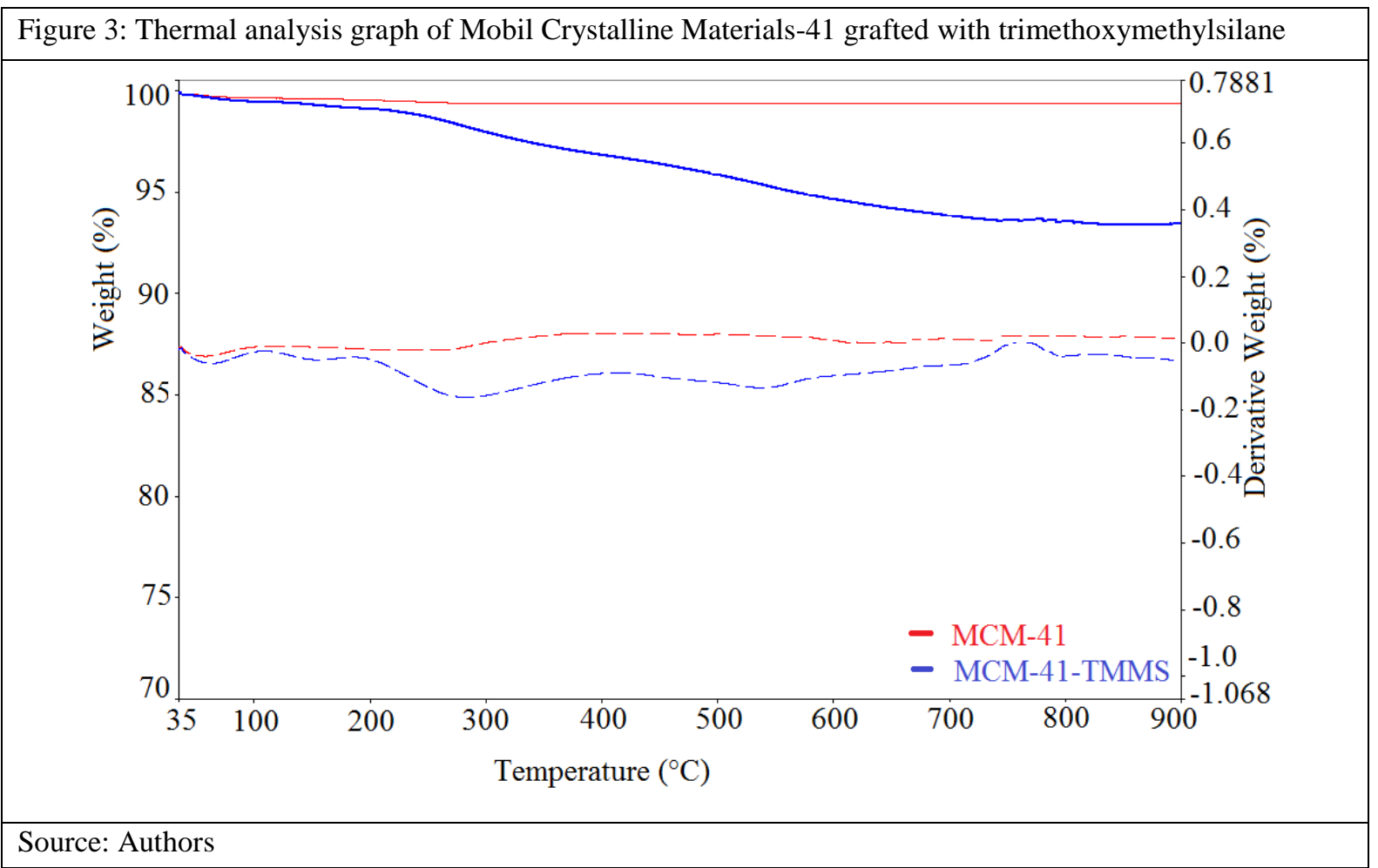

The TG or DTG curves of the samples are shown in Figure 3. For MCM-41, only one weight loss was found to occur between $35{ }^{\circ} \mathrm{C}$ and $105{ }^{\circ} \mathrm{C}$, with the weight loss of $0.65 \%$ due to the removal of physisorbed water on the external surface of the adsorbent. From $105{ }^{\circ} \mathrm{C}$ to $900{ }^{\circ} \mathrm{C} \mathrm{MCM}-41$ presented no obvious weight loss (Taib et al., 2011). The MCM-41-TMMS demonstrated minor weight loss up to $220^{\circ} \mathrm{C}$. From this temperature to $820^{\circ} \mathrm{C}$, a weight loss of $5.6 \%$ was observed due to the decomposition of the TMMS (Deepak et al., 2014).

Table 1: Structural properties of the adsorbents: Mobil Crystalline Materials-41 (MCM-41) alone and with grafting with trimethoxymethylsilane (MCM-41-TMMS)

\begin{tabular}{|c|c|c|}
\hline Adsorbents & $\mathbf{S}_{\text {BET }}\left(\mathbf{m}^{\mathbf{2}} \mathbf{g}^{-1}\right)$ & Pore volume $\left(\mathbf{c m}^{\mathbf{3}} \mathbf{g}^{-1}\right)$ \\
\hline MCM-41 & 939.98 & 0.61 \\
\hline MCM-41-TMMS & 600.87 & 0.47 \\
\hline
\end{tabular}

$\mathrm{S}_{\mathrm{BET}}$ : Specific area surface under Brunaeur-Emmett-Teller theory.

Source: Authors

The textural properties of the samples are listed in Table 1. There was a decrease in both the specific area surface ( $\mathrm{S}_{\mathrm{BET}}$ ) and the pore volumes of the MCM-41 after functionalization (MCM-41-TMMS) that was related to replacing the silanol groups on the silica inner surface with methyl groups.

\section{Conclusion}

In this study, MCM-41 was successfully synthesized from pure silica source. The obtained sample was functionalized with methyl groups to improve surface properties. The characterization of the samples was carried out using different analysis techniques. According to the results of these, the methyl groups were successfully grafted on the sample surface. The $S_{\mathrm{BET}}$ and pore volumes of the MCM-41TMMS were lower after the functionalization. The thermal stability temperature of MCM-41-TMMS in a nitrogen atmosphere was determined at about $220^{\circ} \mathrm{C}$.

\section{References}

Al-Oweini, R., \& El-Rassy, H. (2009). Synthesis and characterization by FTIR spectroscopy of silica aerogels prepared using several $\mathrm{Si}(\mathrm{OR})_{4}$ and R00Si(OR0) $)_{3}$ precursors. Journal of Molecular Structure. 919 (2009) 140-145.

Araki, S., Doi, H., Sano, Y., Shunsuke, T., \& Yoshikazu, M. (2009). Preparation and $\mathrm{CO}_{2}$ adsorption properties of aminopropyl-functionalized mesoporous silica microspheres. Journal of Colloid Interface Science. 339 (2009) 382-389.

Belmabkhout, Y., \& Serna-Guerrero, S. S. (2009). A. Adsorption of $\mathrm{CO}_{2}$ from dry gasses on MCM-41 silica at ambient temperature and high pressure. 1: Pure $\mathrm{CO}_{2}$ adsorption. Chemical Engineering Science, 64 (2009) 3721-3728. 
Belmabkhout, Y., Serna-Guerrero, R., \&Sayari, A. (2010). Adsorption of $\mathrm{CO}_{2}$-Containing gas mixtures over amine-bearing pore-expanded MCM-41 silica: Application for gas purification. Industrial \& Engineering Chemistry Research. 49 (2010) 359-365.

Builes, S., \& Vega, L. F. (2012). Understanding CO2 Capture in amine-functionalized MCM-41 by molecular simulation. The Journal of Physical Chemistry C. 116 (2012) 3017-3024.

Deepak, B. N., Surjyakanta, R., Kulamani, P., \& Bhalchandra, M. B. (2014). Amine functionalized MCM-41 as a green, efficient, and heterogeneous catalyst for the regioselective synthesis of 5-aryl-2-oxazolidinones, from $\mathrm{CO}_{2}$ and aziridines. Applied Catalysis A: General. 469 (2014) 340-349.

Faghihian, H., \& Naghavi, M. (2014). Synthesis of Amine-Functionalized MCM-41 and MCM-48 for removal of heavy metal ions from aqueous solutions. Separation Science and Technology. 49 (2014) 214-220.

Feil, F., Jung, C., Kirstein, J., Michaelis, J., Li, C., Nolde, F., Müllen, K., \& Bräuchle, C. (2009). Diffusional and orientational dynamics of various single terylenediimideconjugates in mesoporous materials. Microporous and Mesoporous Materials. 125 (2009) 70-78.

Gaydhankar, T.R., \& Samuel, Joshi P.N. (2006). Hydrothermal synthesis of MCM-41 using differently manufactured amorphous deoxosilicon sources. Materials Letters. 60 (2006) 957-961.

Gholami, M., Talaie, M.R., Aghamiri, S.F. (2016). $\mathrm{CO}_{2}$ adsorption on amine functionalized MCM-41: Effect of bi-modal porous structure. Journal of the Taiwan Institute of Chemical Engineers. 59 (2016) 205-209.

Kenneth S. W. S. (1998). Adsorption methods for the characterization of porous materials. Advances in Colloid and Interface Science. 76-77 (1998) 3-11.

Kim, J., Kwak, J., Jun, S., \& Ryoo, R. (1995). Ion exchange and thermal stability of MCM-41. Journal of Physical Chemistry. 99 (1995) 16742-16747.

Klinthong, W., Chao K.J., \& Tan, C.S. (2013). $\mathrm{CO}_{2}$ Capture by as-synthesized amine-functionalized mcm-41 prepared through direct synthesis under basic condition. Industrial \& Engineering Chemistry Research. 52(2013) 9834-9842.

Kozhevnikov, I. V., Sinnema, A., Jansen, R. J. J., Pamin,K., \& Bekkum, H.V. (1995). New acid catalyst comprising hereropoly acid on a mesoporous molecular sieve MCM-41. Catalysis Letters, 30 (1995) 241-252.

Liu, Z., Teng, Y., Zhang, K., Chen, H., \& Yang, Y. (2015). $\mathrm{CO}_{2}$ adsorption performance of different amine-based siliceous MCM-41 materials. Journal of Energy Chemistry. 24 (2015) 322-330.

Manzano, M., Aina, V.,Areán, C. O., Balas, F., Cauda, V., Colilla, M., Delgado, M. R., \& Vallet-Regí, M. (2008). Studies on MCM-41 mesoporous silica for drug delivery: Effect of particle morphology and amine functionalization. Chemical Engineering Journal. 137 (2008) 30-37.

Rath, D., Rana, S., \& Parida, K. M. (2014). Organic amine-functionalized silica-based mesoporous materials: an update of syntheses and catalytic applications, An international journal to further the chemical sciences. 4 (2014) 57111-57124.

Selvam, P., Bhatia, S.K., \& Sonwane, C.G. (2001). Recent Advances in processing and characterization of periodic mesoporous MCM-41 silicate molecular sieves. Industrial \& Engineering Chemistry Research, 40 (2001) 3237-3261.

Taib, N. I., Endud, S., \& Katun, N. (2011). Functionalization of mesoporous Si-MCM-41 by grafting with trimethylchlorosilane. International Journal of Chemistry. 3 (1011) 2-10.

Vartuli, J. C., Kresge, C. T., Roth, W. J., Sharon, B. M., Beck, J. S., Schmitt, K.D., Leonowicz, M. E., Lutner, J. D., \& Sheppard, E. W. (1996). Designed synthesis of mesoporous molecular sieve systems using surfactant-directing agents. In Advanced Catalysts and Nanostructured Materials, Academic Press: San Diego.

Xiao-Dong, L., Qing-Zhou, Z., \& Ming-Qiang, Z. (2010). Optical properties of (nanometer MCM-41)-(malachite green) composite materials. Applied Surface Science, 257 (2010) 1134-1140.

Wang, X., Chen, L., \& Guo, Q. (2015). Development of hybrid amine-functionalized MCM-41 sorbents for $\mathrm{CO}_{2}$ capture. Chemical Engineering Journal. 260 (2015) 573-581.

Wu, S., Li, F., Xu, R., Wei, S., \& Li, G. (2010). Synthesis of thiol-functionalized MCM-41 mesoporous silicas and its application in $\mathrm{Cu}$ (II), $\mathrm{Pb}$ (II), $\mathrm{Ag}(\mathrm{I})$, and $\mathrm{Cr}$ (III) removal. Journal of Nanoparticle Research. 12 (2010) 2111-2124.

Yuliarto, B., Zhou, H., Yamada, T., Honma, I., \& Asai, K. (2004). Synthesis of a surface photovoltage sensor using selfordered in-modified MCM-41 films: Enhanced $\mathrm{NO}_{2}$ gas sensing. A European Journal of Chemical Physics and Physical Chemistry, 5 (2004) 261-265.

Zhao, X.S., Lu, G.Q., \& Millar, G.J. (1996). Advances in mesoporous molecular sieve MCM-41. Industrial \& Engineering Chemistry Research, 35 (1996) 2075-2090.

Zhao, X.S., \& Lu, G.Q. (1998). Modification of MCM-41 by surface silylation with trimethylchlorosilane and adsorption study. Journal of Physical Chemistry B. 102 (1998) 1556-1561.

Zhao, X. S., Lu, G. Q., \& Hu, X. (2000). Characterization of the structural and surface properties of chemically modified MCM-41 material. Microporous and Mesoporous Materials. 41 (2000) 37-47 